\title{
Watch what you type: The role of visual feedback from the screen and hands in skilled typewriting
}

\author{
Kristy M. Snyder • Gordon D. Logan • \\ Motonori Yamaguchi
}

Published online: 21 August 2014

(C) The Psychonomic Society, Inc. 2014

\begin{abstract}
Skilled typing is controlled by two hierarchically structured processing loops (Logan \& Crump, 2011): The outer loop, which produces words, commands the inner loop, which produces keystrokes. Here, we assessed the interplay between the two loops by investigating how visual feedback from the screen (responses either were or were not echoed on the screen) and the hands (the hands either were or were not covered with a box) influences the control of skilled typing. Our results indicated, first, that the reaction time of the first keystroke was longer when responses were not echoed than when they were. Also, the interkeystroke interval (IKSI) was longer when the hands were covered than when they were visible, and the IKSI for responses that were not echoed was longer when explicit error monitoring was required (Exp. 2) than when it was not required (Exp. 1). Finally, explicit error monitoring was more accurate when response echoes were present than when they were absent, and implicit error monitoring (i.e., posterror slowing) was not influenced by visual feedback from the screen or the hands. These findings suggest that the outer loop adjusts the inner-loop timing parameters to compensate for reductions in visual feedback. We suggest that these adjustments are preemptive control strategies designed to execute keystrokes more cautiously when visual feedback from the hands is absent, to generate more cautious motor programs when visual feedback from the screen is absent, and to enable enough time for the outer loop to monitor keystrokes when visual feedback
\end{abstract}

K. M. Snyder $(\bowtie) \cdot$ G. D. Logan • M. Yamaguchi

Vanderbilt University, Nashville, TN, USA

e-mail: kristy.m.snyder@vanderbilt.edu

M. Yamaguchi

Edge Hill University, Ormskirk, UK from the screen is absent and explicit error reports are required.

Keywords Hierarchical control $\cdot$ Skilled performance $\cdot$ Motor control · Visual feedback · Typing

Successful motor performance depends on monitoring feedback from our actions and their environmental effects to ensure that goals are met and errors are detected and corrected (Lashley, 1951; Miller, Galanter, \& Pribram, 1960). For novel behaviors, the cognitive system uses explicit processes to monitor feedback consciously. For skilled behaviors, the cognitive system uses explicit processes to monitor some feedback consciously and uses implicit processes to monitor other feedback unconsciously. In the present work, we investigate how the availability of visual feedback influences the explicit and implicit components of skilled typing performance.

Humans are capable of acquiring skill in a great number of motor behaviors, from playing the piano or guitar to playing basketball or golf. Most people are not skilled musicians or athletes, but most Westerners are skilled typists. Today, the average college student receives formal touch-typing training in grade school, hones the typing skill on a laptop computer, and has at least 10 years of typing experience (Logan \& Crump, 2011). As a result, students are able to execute approximately 5-6 keystrokes a second with a high degree of accuracy. The mechanisms that enable such skilled performance become tuned to the kinds of feedback that are typically available: visual feedback from the screen, visual feedback from the hands on the keyboard, and kinesthetic feedback from the hands on the keyboard (Diehl \& Seibel, 1962; Logan \& Crump, 2010; Long, 1976; Rabbitt, 1978; Tapp \& Logan, 2011; West, 1967). Here, we test how removing visual feedback from the screen and hands influences the way that skilled typing is controlled. 


\section{Hierarchical control of skilled typing}

Many researchers have suggested that hierarchically structured cognitive processes control skilled, sequential behaviors, such as typing (e.g., Keele, Cohen, \& Ivry, 1990; Logan \& Crump, 2011; Miller, Galanter, \& Pribram, 1960; Rosenbaum, Cohen, Jax, Weiss, \& van der Wel, 2007; Rumelhart \& Norman, 1982; Salthouse, 1986; Shaffer, 1976; Sternberg, Knoll, \& Turock, 1990). Logan and Crump (2011) proposed a hierarchical, two-loop theory in which two nested control loops govern skilled typewriting (see Fig. 1a). The two loops can be dissociated in a number of ways: Outerloop processes are generally explicit. Inner-loop processes are generally implicit. The outer loop operates at the level of words, the inner loop operates at the level of keystrokes (Crump \& Logan, 2010a; Snyder \& Logan, 2013; Tapp \& Logan, 2011). The outer loop harnesses language comprehension and generation processes to produce a series of words, whereas the inner loop codes motor programs (Keele et al., 1990; Miller et al., 1960; Snyder \& Logan 2014) to produce a series of keystrokes. The outer loop monitors visual feedback from the screen to ensure that the words are produced accurately, and the inner loop monitors kinesthetic feedback from the hands to ensure that the keystrokes are executed accurately (Crump \& Logan, 2010b; Logan \& Crump, 2010).

Many studies have demonstrated dissociations between the two loops (e.g., Abrahamse, Ruitenberg, de Kleine, \& Verwey, 2013; Crump \& Logan, 2010a, b; Logan \& Crump,

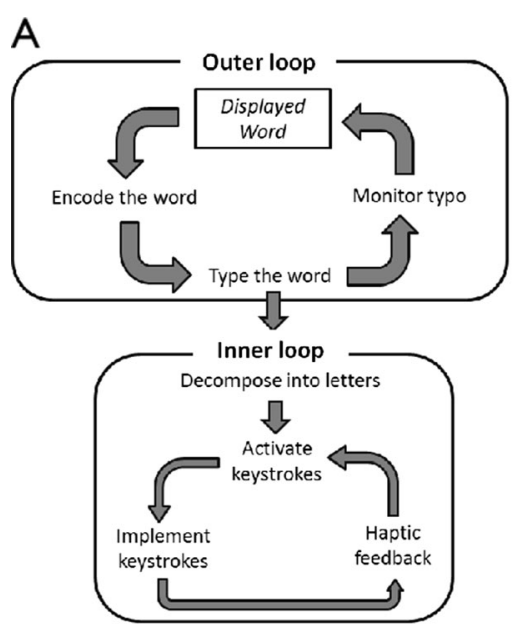

B

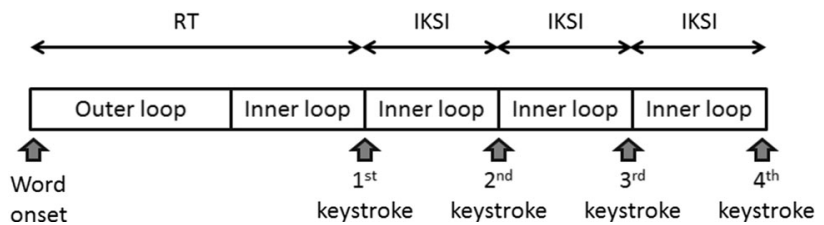

Fig. 1 (a) Illustration of the two-loop theory of skilled typewriting. (b) Latency measures from the discrete typing task.
2009, 2010; Snyder \& Logan, 2013; Tapp \& Logan, 2011; Verwey, 1999; Yamaguchi \& Logan, 2014; Yamaguchi, Logan, \& Li 2013b), but few studies have addressed how they work together to control typing (Yamaguchi, Crump, \& Logan 2013a). The inner loop is subordinate to the outer loop (Logan \& Crump, 2011), so inner-loop processes are subject to outer-loop processes. The outer loop specifies the word that should be typed, and the inner loop identifies and orders the corresponding keystrokes. The outer loop also adjusts the timing parameters within which the inner loop must operate to compensate for atypical typing conditions: For instance, the typing rate can approximate an experimentally manipulated tempo (Yamaguchi \& Logan 2014) and can slow to allow enough time to monitor individual keystrokes explicitly (Crump \& Logan 2010b; Snyder \& Logan, 2013; Tapp \& Logan, 2011). The purpose of the present study was to test whether the outer loop will also adjust inner-loop timing parameters to compensate for the absence of visual feedback.

\section{The present study}

In the present study, we used a discrete typing task in which skilled typists typed single target words that were displayed on a computer screen one at a time. We manipulated the availability of visual feedback on the screen: Typists' responses were either echoed on the screen, so the letter that corresponded to each keystroke appeared below the target word, or the echoes were withheld, so that nothing appeared on the screen. We also manipulated the visual feedback from the hands: Typists' hands and the keyboard were either visible or covered with a box. We thus created four conditions, one for each combination of keystroke echoes present or absent and hands visible or covered. These conditions were blocked, and typists were told what combination of feedback would be available before they began each block.

We assessed the inner- and outer-loop processes with four measures: reaction time (RT), interkeystroke interval (IKSI), explicit error report, and posterror slowing. RT is the latency between stimulus onset and the first keystroke, which reflects the time the outer loop takes to encode a word plus the time the inner loop takes to program the corresponding motor program and to execute the first keystroke (see Fig. 1b). IKSI is the average time between successive keystrokes, which reflects the time the inner loop takes to execute each keystroke. Explicit error report is a typist's judgment of typing accuracy, reflecting error detection in the outer loop. Posterror slowing is the extended interkeystroke interval following an error (Rabbitt, 1966), which reflects error detection in the inner loop (Crump \& Logan, 2013; Gordon \& Soechting, 1995; Yamaguchi \& Logan, 2014). 


\section{Experiment 1}

In the first experiment, we manipulated the visual feedback from the screen by echoing or not echoing the keystrokes that typists typed on the screen below the word that they copied. We manipulated the visual feedback from the hands by covering or not covering the typists' hands and keyboard with a box. There were four blocks of trials, one for each combination of keystrokes echoed versus not echoed and hands covered versus not covered. We assessed how visual feedback influenced the generation of the motor program by measuring $\mathrm{RT}$, the implementation of the motor program by measuring IKSI, and implicit error detection by measuring posterror slowing.

If the outer loop adjusts inner-loop parameters to compensate for the availability of visual feedback from the screen, RTs should be affected. RT reflects the time it takes the outer loop to encode the word and the time it takes the inner loop to generate a motor program and execute the first keystroke. When keystrokes are echoed on the screen, the inner loop can afford to generate motor programs rapidly, because little cost is associated with correcting an error: The outer loop sees the error and specifies how the inner loop should correct it. When keystrokes are not echoed on the screen, this strategy is not possible. The outer loop may compensate by instructing the inner loop to take more time, to generate motor programs more cautiously. If so, RTs should be longer when response echoes are absent than when they are present.

Visual feedback from the screen should not affect IKSI, which reflects the time it takes the inner loop to execute keystrokes. The inner loop typically uses kinesthetic feedback from the hands to monitor keystrokes implicitly (Crump \& Logan, 2010a; Logan \& Crump, 2010, 2011). Therefore, the presence or absence of response echoes should not influence the rate at which keystrokes are executed.

Visual feedback from the hands should influence IKSI. Visual feedback from the hands on the keyboard is not necessary for skilled typing (Tapp \& Logan, 2011), but it is helpful for aligning the fingers to the keys (Tapp \& Logan, 2011; see also Long, 1976) and for correcting the trajectories of finger movements (Fautrelle \& Bonnetblanc, 2012). Therefore, keystrokes should be less accurate when the hands are covered than when they are visible. There is a speed-accuracy trade-off in typing (Yamaguchi et al. 2013a, b), so the outer loop may compensate by slowing the rate at which the inner loop executes keystrokes. Thus, IKSIs should be longer when the hands are covered than when they are visible.

Visible feedback from the screen and hands should not influence posterror slowing, which reflects error detection in the inner loop. The inner loop ensures typing accuracy by implicitly monitoring kinesthetic feedback from the hands on the keyboard (Logan \& Crump, 2011). Therefore, posterror slowing should not differ when response echoes are present or absent. Posterror slowing should also not differ when the hands are visible or covered.

\section{Method}

Subjects Twenty-four touch-typists were recruited from the Vanderbilt University community; they reported being able to touch-type at least 40 words per minute (WPM). The typists were also required to have had formal typing training: 13 had completed an elementary school course, eight had completed a middle school course, two had completed a high school course, and one had completed a home computer program. Typing skill was verified with a test in which the typists typed short paragraphs (Logan \& Zbrodoff, 1998). Their mean typing speed was $76.5 \mathrm{WPM}$ (range $=41.8-117.7 \mathrm{WPM}$ ) and mean accuracy was $91.9 \%$ (range $=81.5 \%-96.5 \%$ ). All typists were compensated with course credits or were paid $\$ 12$ for $1 \mathrm{~h}$ of participation. All of the typists had normal or corrected-to-normal vision and spoke English as a first language.

Apparatus and stimuli The experiment was conducted individually in a cubicle under normal fluorescent lighting on a Dell Vostro 200 personal computer. Typists sat approximately $55-60 \mathrm{~cm}$ in front of a 19-in. color monitor and made responses on a standard QWERTY keyboard. The stimuli were five-letter words, presented by an experimental program written by METACARD. A pool of 150 five-letter words was compiled from the MRC Psycholinguistic Database (Wilson, 1987). The mean word frequency per million words was 53.1 (range $=0.87-488.2$; Davies, 2008). The words were presented in black 44-pt. Helvetica font within a $24.1 \times 19.7 \mathrm{~cm}$ gray window that was opened by the experimental program. In some of the trials, typists' hands and the keyboard were covered by a $10.8 \times 27.9 \times 44.5 \mathrm{~cm}$ box that did not constrain hand movements.

Procedure Each typist completed four blocks of trials, in which two variables were manipulated in a factorial manner. The first factor was the visibility of the hands interacting with the keyboard. Typists' hands and the keyboard were visible in the keyboard visible condition and were covered by a box in the keyboard covered condition. The second factor was the presence of typing response echoes. Letters typed by the typists were displayed on the computer screen $6.4 \mathrm{~cm}$ below the target word in the echo-present condition and were not displayed on the screen in the echo-absent condition. Each block consisted of 150 trials. All stimuli in the pool were presented in a random order in each block. The order of the four blocks was counterbalanced using a Latin square design.

Each trial began with a $500 \mathrm{~ms}$ blank screen, followed by the presentation of a fixation cross that appeared $6.4 \mathrm{~cm}$ from 
the top of the window for $500 \mathrm{~ms}$. After a second $500 \mathrm{~ms}$ blank screen interval, a target word appeared at the center of screen. Typists were to type the target word as quickly and as accurately as possible and then to press the spacebar. The backspace key was disabled so that the typists were unable to correct errors. Trials were considered correct only if all letters were typed in the correct order. After typists had completed the four blocks of trials, they performed a typing test, which assessed their typing skill. The keyboard was visible and typed letters were echoed during the typing test. Each session lasted approximately $1 \mathrm{~h}$.

\section{Results and discussion}

Measures and analyses We calculated the mean RT, IKSI, error rate, and posterror slowing for each typist. The RT was the interval between the stimulus onset and when the first keystroke was executed. An ANOVA comparing correct and error RTs revealed no significant difference between correct and error trials and no interactions, so we report analyses that were conducted on RTs from correct trials only. The average RT for each condition is presented in Table 1 and plotted above Position 1 in Fig. 2a. The IKSI is the slope of the linear function that relates each keystroke's timing to its position in a word (see Fig. 2a), reported as time per keystroke (i.e., IKSI = $\mathrm{ms} /$ keystroke). IKSI was calculated from the interkeystroke intervals of correct trials only. The interkeystroke intervals from error trials were analyzed separately in the posterror slowing analyses. The error rate was the percentage of trials in which at least one typing error was committed. The RTs and IKSIs from correct trials and arcsine-transformed error rates were submitted to 2 (keyboard: visible vs. covered) $\times 2$ (echo: present vs. absent) analyses of variance (ANOVAs). A summary table for the ANOVAs is presented in Table 2.

Posterror slowing was the prolonged interkeystroke interval that followed an error relative to the interkeystroke interval that had preceded the error. The error keystroke (E) was defined as the keystroke that corresponded to the first letter typed that deviated from the letter that was supposed to be

Table 1 Mean reaction times for the first keystroke (RT), mean interkeystroke intervals (IKSI), and mean error rates (ER) for each condition for Experiment 1

\begin{tabular}{llllll}
\hline Condition & \multicolumn{3}{l}{ Measure } & & \\
\cline { 1 - 2 } \cline { 5 - 6 } Keyboard & \multirow{2}{nnnn}{\cline { 5 - 6 } Echo } & & RT (ms) & IKSI (ms/keystroke) & ER (\%) \\
\hline Visible & Present & 613 & 138 & 11.7 \\
Visible & Absent & 627 & 142 & 9.2 \\
Covered & Present & 623 & 150 & 15.7 \\
Covered & Absent & 636 & 154 & 17.0 \\
\hline
\end{tabular}

A

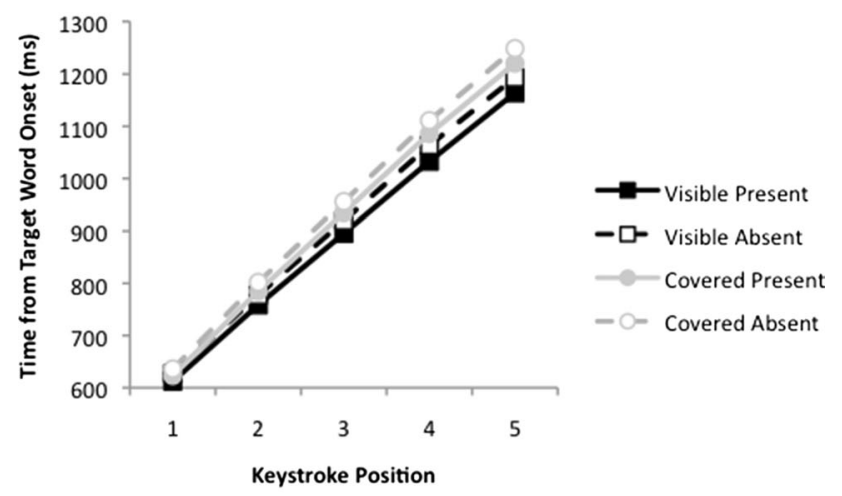

B

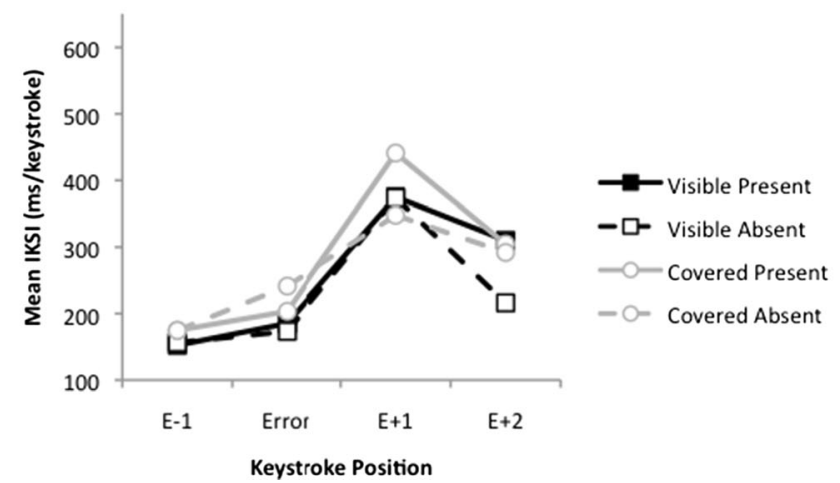

Fig. 2 (a) Average latency measures for correct responses in Experiment 1. (b) Mean interkeystroke intervals per letter for the keystroke preceding an error $(E-1)$, the error keystroke $(E)$, and the two keystrokes following the error $(E+1, E+2)$ for Experiment 1. Data from the keyboard-visible trials are represented with black lines, and data from the keyboardcovered trials are represented with gray lines. Data from echo-present trials are represented with solid lines, and data from echo-absent trials are represented with dashed lines.

typed, regardless of the type of error committed (e.g., substitutions, omissions, transpositions, etc.). The mean IKSI for the keystroke before an error $(E-1)$, the error keystroke $(E)$, and the two keystrokes following an error $(\mathrm{E}+1$ and $\mathrm{E}+2)$ are displayed in Fig. $2 b$. The IKSI for error trials was estimated by calculating the differences between the times at which those keystrokes occurred (i.e., IKSI $=\mathrm{RT}_{N}-\mathrm{RT}_{N-1}$ ). Posterror slowing was assessed with a 2 (keyboard: visible vs. covered) $\times 2$ (echo: present vs. absent) $\times 2$ (position: $\mathrm{E}-1$ vs. $\mathrm{E}+1$ ) ANOVA. The summary table is presented in Table 3 .

Visual feedback from the screen If the outer loop adjusts inner-loop parameters to compensate for the availability of visual feedback from the screen, RTs should be longer when response echoes are absent than when they are present. As is indicated in Table 2, the RT was significantly longer when echoes were absent $(M=632 \mathrm{~ms})$ than when they were present $(M=618 \mathrm{~ms})$. The inner loop relies on kinesthetic feedback from the hands (Logan \& Crump, 2011), so visual feedback 
Table 2 Summary table for the 2 (keyboard: visible vs. covered) $\times 2$ (echo: present vs. absent) analyses of variance conducted on reaction times to the first letter (RT), interkeystroke intervals (IKSI), and arcsine-corrected error rates (ER) data from Experiment 1

\begin{tabular}{|c|c|c|c|c|c|c|c|c|c|}
\hline \multirow[b]{2}{*}{ Effect } & \multicolumn{3}{|l|}{ RT } & \multicolumn{3}{|l|}{ IKSI } & \multicolumn{3}{|l|}{ ER } \\
\hline & $M S E$ & $F$ & $\eta_{\mathrm{p}}^{2}$ & $M S E$ & $F$ & $\eta_{\mathrm{p}}^{2}$ & $M S E$ & $F$ & $\eta_{\mathrm{p}}^{2}$ \\
\hline Keyboard (K) & $2,469.5$ & $<1$ & .039 & 711.6 & $4.7^{*}$ & .169 & .09 & $6.7^{*}$ & .226 \\
\hline Echo (E) & 675.2 & $6.8^{*}$ & .229 & 146.8 & 2.7 & .103 & .02 & 1.5 & .060 \\
\hline $\mathrm{K} \times \mathrm{E}$ & 445.1 & $<1$ & .003 & 39.1 & $<1$ & .000 & .01 & 3.5 & .131 \\
\hline
\end{tabular}

Degrees of freedom for each effect $=1,23 ;{ }^{*} p<.05$

from the screen should not influence the IKSI, error rates, or posterror slowing. IKSIs did not differ significantly between the echo-absent ( $M=148 \mathrm{~ms} /$ keystroke) and echo-present ( $M$ $=144 \mathrm{~ms} /$ keystroke) conditions. Error rates also did not differ significantly between the echo-absent $(M=13.2 \%)$ and echopresent $(M=13.7 \%)$ conditions. The posterror slowing analysis (see Table 3) revealed a significant main effect of position, such that the IKSI of the keystroke that followed the error ( $M=385 \mathrm{~ms} /$ keystroke) was significantly longer than the IKSI of the keystroke that preceded the error $(M=165 \mathrm{~ms} /$ keystroke). This finding was indicative of posterror slowing. We found no significant effect of echo or significant interaction between position and echo. These findings suggest that posterror slowing was not influenced by the presence or absence of response echoes.

Visual feedback from the hands and keyboard The two-loop theory states that neither loop principally relies on visual feedback from the hands and keyboard (Crump \& Logan, 2010a; Logan \& Crump, 2010, 2011), so covering the hands and keyboard should not influence RTs, IKSIs, or posterror slowing. However, the inner loop uses visual feedback from the hands to align the fingers with the keys (Long, 1976) and guide keystroke trajectories (Fautrelle \& Bonnetblanc, 2012).

Table 3 Summary table for the 2 (keyboard: visible vs. covered) $\times 2$ (echo: present vs. absent) $\times 2$ (position: $\mathrm{E}-1$ vs. $\mathrm{E}+1$ ) ANOVA conducted on the IKSIs of the keystrokes that preceded and followed error keystrokes for Experiment 1

\begin{tabular}{llll}
\hline Effect & MSE & $F$ & $\eta_{\mathrm{p}}^{2}$ \\
\hline Keyboard (K) & $14,656.2$ & 1.3 & .055 \\
Echo (E) & $21,755.8$ & 1.1 & .047 \\
Position (P) & $49,683.6$ & $46.7^{*}$ & .670 \\
$\mathrm{~K} \times \mathrm{E}$ & $16,467.3$ & 1.7 & .068 \\
$\mathrm{~K} \times \mathrm{P}$ & $10,434.9$ & $<1$ & .000 \\
$\mathrm{E} \times \mathrm{P}$ & $20,629.3$ & 1.4 & .058 \\
$\mathrm{~K} \times \mathrm{P} \times \mathrm{E}$ & $17,848.5$ & 1.3 & .053 \\
\hline
\end{tabular}

Degrees of freedom for each effect $=1,23 ;{ }^{*} p<.05$
Therefore, the outer loop might adjust the inner-loop parameters to favor accuracy over speed (Yamaguchi et al. 2013a). As a result, IKSIs should be longer when the hands and keyboard are covered than when they are visible. As is indicated in Table 2, IKSIs were significantly longer when the hands and keyboard were covered $(M=152 \mathrm{~ms} /$ keystroke $)$ than when they were visible $(M=140 \mathrm{~ms} /$ keystroke).

There was a similar effect on RTs. The time that it takes the inner loop to execute the first keystroke is included in the RT, so any variable that affects IKSI should also affect RT by the same amount. The variability in RT was larger than the variability in IKSI because RT reflects both outer- and inner-loop processes, whereas IKSI reflects only inner-loop processes (Logan \& Crump, 2011). Thus, the variability of RT might wash out the statistical significance of the effect. Indeed, RTs were longer when the hands and keyboard were covered $(M=$ $630 \mathrm{~ms})$ than when they were visible $(M=620 \mathrm{~ms})$ by an amount similar to the effect in IKSI ( $10 \mathrm{~ms}$ in RT vs. $12 \mathrm{~ms} /$ keystroke in IKSI). Yet, the effect was not statistically significant (see Table 2).

Keyboard visibility did affect error rates (see Table 2), which were significantly lower when the hands and keyboard were visible $(M=10.5 \%)$ than when they were covered $(M=$ $16.4 \%$ ). However, keyboard visibility did not affect posterror slowing (see Table 3), and no significant effect of keyboard or significant interaction between position and keyboard emerged.

\section{Conclusions}

The results of Experiment 1 supported the notion that the outer loop adjusts the parameters of inner-loop processing to compensate for the availability of visual feedback. Visual feedback from the screen influenced RT. We suggest that motor programs were planned more cautiously when typing responses were not echoed on the screen. Visual feedback from the hands influenced IKSI and the IKSI component of RT. Because the time that it takes to execute the first keystroke is included in the RT, visual feedback from the hands affected 
RT by the same magnitude as the effect on IKSI: The effect on IKSI was $12 \mathrm{~ms} /$ keystroke, and the effect on RT was $10 \mathrm{~ms}$. We suggest that keystroke execution was disrupted when typists could not rely on vision to align their fingers with the keys (Long, 1976) or to guide keystroke trajectories (Fautrelle \& Bonnetblanc, 2012). To compensate, the outer loop adjusted the inner-loop timing parameters to allow for slower, and more accurate, keystroke execution.

Visual feedback from the screen or from the hands did not influence implicit error detection. Researchers have suggested that implicit error detection relies on haptic feedback to the fingers (Gordon \& Soechting, 1995; Logan \& Crump, 2010), and the present results confirmed that it does not depend on visual feedback. Skilled typing also depends on explicit error detection that relies on visual feedback from the screen (Rabbitt, 1978). In Experiment 2, we tested explicit and implicit error detection.

\section{Experiment 2}

In Experiment 2, we investigated how visual feedback influences outer- and inner-loop processes when typists are required to provide explicit error reports. This requirement should provide more incentive for the outer loop to modify inner-loop timing (Snyder \& Logan, 2013). We used the same discrete typing task as in Experiment 1 but prompted the typists to report whether or not they typed the word correctly after they finished typing each word.

Visual feedback from the screen should influence explicit error reports. Explicit error detection is associated with outerloop processing (Crump \& Logan, 2013; Logan \& Crump, 2011). The outer loop ensures accurate typing by explicitly monitoring visual feedback from the screen (Crump \& Logan, 2010a; Logan \& Crump, 2010, 2011). This strategy is not possible when keystrokes are not echoed on the screen. Thus, explicit error reports should be less accurate when echoes are absent than when they are present (Logan \& Crump, 2010).

As in Experiment 1, visual feedback from the screen should influence RT, and neither source of visual feedback should influence posterror slowing. However, unlike Experiment 1, visual feedback from the screen should influence IKSI in Experiment 2. When typists are required to give explicit error reports and response echoes are absent, the outer loop must monitor keystrokes. Keystrokes are typically executed too rapidly to be monitored explicitly (Keele, 1968; Keele \& Posner, 1968; Snyder \& Logan, 2013), so the outer loop compensates by slowing the rate at which the inner loop executes keystrokes so that explicit monitoring is possible (Logan \& Crump, 2009; Snyder \& Logan, 2013; Tapp \& Logan, 2011). Thus, IKSI will be longer when response echoes are absent than when they are present.

\section{Method}

Subjects A new group of 24 touch-typists were recruited from the same subject pool as in Experiment 1. Again, the typists were required to have had formal touch-typing training: 11 had completed an elementary school course, nine had completed a middle school course, three had completed a high school course, and one had completed a home computer program. Their mean typing speed, as assessed by the same typing test as in Experiment 1, was 83.8 WPM (range $=49.9$ 124.6 WPM), and their mean accuracy was $93.9 \%$ (range = $88.1 \%-98.2 \%)$.

Apparatus, stimuli, and procedure The apparatus and stimuli were the same as in Experiment 1, except that a probe screen was inserted after typists typed the target word and pressed the space bar on each trial. The probe presented the question, "Did you make an error," and typists had $2 \mathrm{~s}$ to type "Y" if they had made an error during the previous trial, or to type " $\mathrm{N}$ " if they had not. The next trial started $2 \mathrm{~s}$ after the probe was presented.

\section{Results and discussion}

Measures and analyses RTs, IKSIs, error rates, and posterror slowing were calculated and analyzed in the same manner as was described for Experiment 1 . The means across typists are presented in Table 4, and the mean times at which each keystroke occurred are displayed in Fig. 3a. The RTs and IKSIs for correct trials and arcsine-corrected error rates were subjected to 2 (keyboard: visible vs. covered) $\times 2$ (echo: present vs. absent) ANOVAs. A summary table for the ANOVAs appears in Table 5. Posterror slowing was assessed with a 2 (keyboard: visible vs. covered) $\times 2$ (echo: present vs. absent) $\times 2$ (position: $\mathrm{E}-1$ vs. $\mathrm{E}+1$ ) ANOVA, and the summary table appears in Table 6. The mean IKSIs for E 1, E, E + 1, and E +2 are displayed in Fig. 3b.

Explicit error detection was measured by calculating the percentage of trials in which typists reported correct

Table 4 Mean reaction times for the first keystroke (RT), mean interkeystroke intervals (IKSI), and mean error rates (ER) for each condition in Experiment 2

\begin{tabular}{lllll}
\hline Condition & \multicolumn{5}{c}{ Measure } \\
\hline Keyboard & Echo & RT (ms) & IKSI (ms/keystroke) & ER (\%) \\
Visible & Present & 617 & 127 & 8.8 \\
Visible & Absent & 645 & 143 & 4.8 \\
Covered & Present & 631 & 136 & 9.9 \\
Covered & Absent & 650 & 152 & 10.3 \\
\hline
\end{tabular}


A

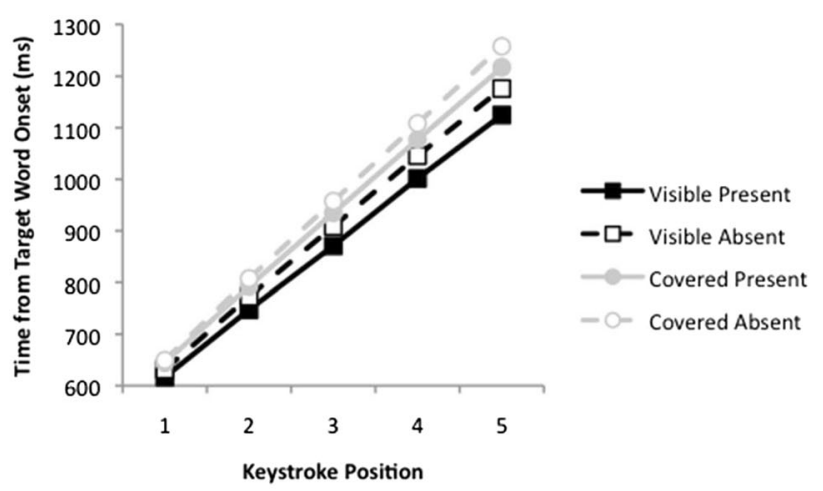

B

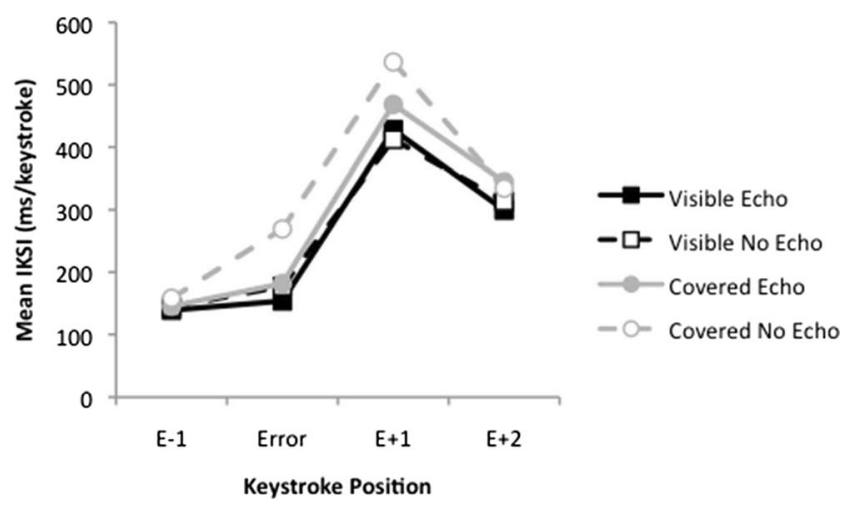

Fig. 3 (a) Average latency measures for correct responses in Experiment 2. (b) Mean interkeystroke intervals per letter for the keystroke preceding an error $(\mathrm{E}-1)$, the error keystroke $(\mathrm{E})$, and the two keystrokes following the error $(E+1, E+2)$ for Experiment 2. Data from keyboard-visible trials are represented with black lines, and data from keyboard-covered trials are represented with gray lines. Data from echo-present trials are represented with solid lines, and data from echo-absent trials are represented with dashed lines

performance when their performance actually was correct and the percentage of trials in which typists reported making an error when they actually made an error. The mean percentages for correct and error responses are presented in Table 7. A 2 (keyboard: visible vs. covered) $\times 2$ (echo: present vs. absent $) \times$ 2 (response: error vs. correct) ANOVA was conducted on the explicit report accuracies, with a summary table presented in Table 8.
Visual feedback from the screen As in Experiment 1, RTs were significantly longer when keystroke echoes were absent $(M=648 \mathrm{~ms})$ than when they were present $(M=624 \mathrm{~ms})$. However, unlike in Experiment 1, IKSIs were also significantly longer when keystroke echoes were absent $(M=$ $148 \mathrm{~ms} /$ keystroke) than when they were present $(M=$ $132 \mathrm{~ms} /$ keystroke). Also unlike in Experiment 1, error rates were significantly higher when keystroke echoes were present $(M=9.3 \%)$ than when they were absent $(M=7.6 \%)$. The significant interaction between keyboard and echo for error rates (see Table 5), combined with the IKSI results, is consistent with the notion that the outer loop adapted to the availability of visual feedback by adjusting the inner-loop parameters. As in Experiment 1, the IKSI of the keystroke that followed an error $(M=438 \mathrm{~ms} /$ keystroke) was significantly longer than the IKSI of the keystroke that preceded an error ( $M=153 \mathrm{~ms} /$ keystroke), indicating posterror slowing. As in Experiment 1, posterror slowing was not significantly affected by echo (see Table 6).

Overall, the explicit error report analysis (see Table 7) indicated that typists detected correct performance on nearly all of the trials in which their performance was correct $(M=$ $99.0 \%)$ and detected errors less often $(M=75.7 \%)$. As is indicated in Table 8, error detection accuracy was higher when keystrokes were echoed $(M=89.4 \%)$ than when they were $\operatorname{not}(M=62.1 \%)$.

Visual feedback from the hands and keyboard As in Experiment 1 , IKSIs were significantly longer when the hands and keyboard were covered ( $M=144 \mathrm{~ms} /$ keystroke) than when they were visible ( $M=135 \mathrm{~ms} /$ keystroke). Again, the effect on IKSI was also evident in RT. RTs were longer when the hands and keyboard were covered $(M=641 \mathrm{~ms})$ than when they were visible ( $M=631 \mathrm{~ms})$, by an amount similar to the effect in IKSI (10 ms in RT vs. 9 ms/keystroke in IKSI), but this difference was not statistically significant (see Table 5). Posterror slowing was not significantly affected by covering the keyboard; the interaction between position and hand was not significant (see Table 6). Explicit error reports were not significantly affected by covering the keyboard, either (see Table 8).

Table 5 Summary table for 2 (keyboard: visible vs. covered) $\times 2$ (echo: present vs. absent) analyses of variance conducted on reaction times to the first letter (RT), interkeystroke intervals (IKSI), and arcsine-corrected error rate (ER) data from Experiment 2

\begin{tabular}{|c|c|c|c|c|c|c|c|c|c|}
\hline \multirow[b]{2}{*}{ Effect } & \multicolumn{3}{|l|}{ RT } & \multicolumn{3}{|l|}{ IKSI } & \multicolumn{3}{|l|}{ ER } \\
\hline & $M S E$ & $F$ & $\eta_{\mathrm{p}}{ }^{2}$ & $M S E$ & $F$ & $\eta_{\mathrm{p}}^{2}$ & MSE & $F$ & $\eta_{\mathrm{p}}^{2}$ \\
\hline Keyboard (K) & $1,018.6$ & 2.3 & .089 & 212.9 & $9.1^{*}$ & .284 & .08 & 2.2 & .089 \\
\hline Echo $(\mathrm{E})$ & $2,345.7$ & $5.4^{*}$ & .190 & 216.1 & $27.3^{*}$ & .542 & .03 & $6.3^{*}$ & .216 \\
\hline $\mathrm{K} \times \mathrm{E}$ & 973.1 & $<1$ & .024 & 71.7 & $<1$ & .001 & .03 & $4.3^{*}$ & .157 \\
\hline
\end{tabular}

Degrees of freedom for each effect $=1,23 ;{ }^{*} p<.05$ 
Table 6 Summary table for the 2 (keyboard: visible vs. covered) $\times 2$ (echo: present vs. absent) $\times 2$ (position: $E-1$ vs. $E+1)$ analysis of variance conducted on the IKSIs of the keystrokes that preceded and followed error keystrokes for Experiment 2

\begin{tabular}{llll}
\hline Effect & $M S E$ & $F$ & $\eta_{\mathrm{p}}{ }^{2}$ \\
\hline Keyboard (K) & $16,213.4$ & 5.2 & .185 \\
Echo (E) & $13,673.1$ & 3.2 & .123 \\
Position (P) & $29,769.2$ & $30.7^{*}$ & .571 \\
$\mathrm{~K} \times \mathrm{E}$ & $19,332.9$ & $<1$ & .004 \\
$\mathrm{~K} \times \mathrm{P}$ & $8,510.4$ & $<1$ & .016 \\
$\mathrm{E} \times \mathrm{P}$ & $19,827.6$ & 1.4 & .057 \\
$\mathrm{~K} \times \mathrm{P} \times \mathrm{E}$ & $13,105.5$ & 1.3 & .103 \\
\hline
\end{tabular}

Degrees of freedom for each effect $=1,23 ;{ }^{*} p<.05$

Effect of explicit monitoring The purpose of the present work was to assess whether the outer loop adjusts the inner-loop timing parameters to compensate for the availability of visual feedback. Therefore, we had to determine whether outer- or inner-loop processes are affected by visual feedback. To do so, we conducted 2 (keyboard: visible vs. covered) $\times 2$ (echo: present vs. absent) $\times 2$ (measure: RT vs. IKSI) ANOVAs for the two experiments separately (see Table 9). Interactions between measure and keyboard or echo would indicate a selective effect of feedback on the outer loop, if the effect was stronger for RT than for IKSI. Null interactions between measure and keyboard or echo would indicate a selective effect of feedback on the inner loop, prolonging IKSI and the inner-loop component of RT by the same amount. For Experiment 1, the interaction between echo and measure was significant, indicating stronger effects on RT than on IKSI. This suggests that echo affected the outer loop or an innerloop process that is not reflected in IKSI, perhaps prolonging the time that it took to generate a motor program. For Experiment 2, the interaction between echo and measure was not significant, suggesting that echo affected an innerloop process that is reflected in both RT and IKSI, as the aforementioned analysis of IKSIs indicated.

The interaction between echo and measure was significant in Experiment 1 but not in Experiment 2, suggesting that the

Table 7 Mean percentages of correct error reports in Experiment 2 for error responses and correct responses

\begin{tabular}{|c|c|c|c|}
\hline \multicolumn{2}{|l|}{ Condition } & \multicolumn{2}{|l|}{ Response } \\
\hline Keyboard & Echo & Error $(\%)$ & Correct $(\%)$ \\
\hline Visible & Present & 88.7 & 99.6 \\
\hline Visible & Absent & 63.3 & 99.2 \\
\hline Covered & Present & 90.0 & 99.3 \\
\hline Covered & Absent & 60.9 & 97.8 \\
\hline
\end{tabular}

Table 8 Summary table for the 2 (keyboard: visible vs. covered) $\times 2$ (echo: present vs. absent) $\times 2$ (response: error vs. correct) analysis of variance conducted on the explicit error reports in Experiment 2

\begin{tabular}{llll}
\hline Effect & MSE & $F$ & $\eta_{\mathrm{p}}{ }^{2}$ \\
\hline Keyboard (K) & 129.6 & $<1$ & .008 \\
Echo (E) & 227.6 & $41.8^{*}$ & .645 \\
Response (R) & 273.7 & $94.7^{*}$ & .805 \\
$\mathrm{~K} \times \mathrm{E}$ & 120.2 & $<1$ & .024 \\
$\mathrm{~K} \times \mathrm{R}$ & 138.5 & $<1$ & .000 \\
$\mathrm{E} \times \mathrm{R}$ & 218.9 & $37.9^{*}$ & .622 \\
$\mathrm{~K} \times \mathrm{E} \times \mathrm{R}$ & 127.7 & $<1$ & .006 \\
\hline
\end{tabular}

Degrees of freedom for each effect $=1,23 ;{ }^{*} p<.05$

outer loop compensated for reductions in visual feedback differently in each experiment. To test whether this was the case, we conducted a 2 (keyboard: visible vs. covered) $\times 2$ (echo: present vs. absent) ANOVA with Experiment as a between-subjects factor on the RT and IKSI data (see Table 10). For RT, the interaction between echo and experiment was not significant, which suggests that echo influenced the generation of motor programs in the same way for both experiments. For IKSI, the interaction between echo and experiment was significant, which suggests that echo influenced keystroke execution more in Experiment 2 than in Experiment 1. Thus, when explicit error detection is required, the outer loop compensates for the lack of visual feedback from the screen by monitoring keystrokes explicitly, adjusting the inner-loop timing parameters to allow enough time to discover what the fingers are doing (Snyder \& Logan, 2013).

To determine whether the requirement to give explicit error reports influenced implicit error detection in the inner loop, we conducted a 2 (keyboard: visible vs. covered) $\times 2$ (echo: present vs. absent) $\times 2$ (position: $\mathrm{E}-1$ vs. $\mathrm{E}+1$ ) ANOVA with Experiment as a between-subjects factor. The Position $\times$ Experiment interaction was not significant, $F(1,44)=$

Table 9 Summary table for the 2 (keyboard: visible vs. covered) $\times 2$ (echo: present vs. absent) $\times 2$ (measure: RT1 vs. IKSI) analysis of variance conducted for both experiments

\begin{tabular}{|c|c|c|c|c|c|c|}
\hline \multirow[b]{2}{*}{ Effect } & \multicolumn{3}{|c|}{ Experiment 1} & \multicolumn{3}{|c|}{ Experiment 2} \\
\hline & $M S E$ & $F$ & $\eta_{\mathrm{p}}{ }^{2}$ & $M S E$ & $F$ & $\eta_{\mathrm{p}}{ }^{2}$ \\
\hline Keyboard (K) & $2,701.2$ & 2.1 & .083 & 686.2 & $6.2^{*}$ & .428 \\
\hline Echo $(\mathrm{E})$ & 566.4 & $6.8^{*}$ & .228 & $1,566.2$ & $11.4^{*}$ & .723 \\
\hline Measure (M) & $6,385.7$ & $1,725.3^{*}$ & .987 & $5,661.5$ & $2,085.7^{*}$ & .010 \\
\hline $\mathrm{K} \times \mathrm{E}$ & 257.8 & $<1$ & .003 & 556.1 & $<1$ & .020 \\
\hline $\mathrm{K} \times \mathrm{M}$ & 473.9 & $<1$ & .004 & 545.3 & $<1$ & .167 \\
\hline $\mathrm{E} \times \mathrm{M}$ & 255.6 & $4.5^{*}$ & .165 & 995.6 & $<1$ & .355 \\
\hline $\mathrm{K} \times \mathrm{E} \times \mathrm{M}$ & 226.4 & $<1$ & .003 & 488.6 & $<1$ & .000 \\
\hline
\end{tabular}

Degrees of freedom for each effect $=1,23 ;{ }^{*} p<.05$ 
Table 10 Summary table for the 2 (keyboard: visible vs. covered) $\times 2$ (echo: present vs. absent) analyses of variance with Experiment as a between-subjects factor conducted on the raw RT and IKSI data

\begin{tabular}{|c|c|c|c|c|c|c|}
\hline \multirow[b]{2}{*}{ Effect } & \multicolumn{3}{|l|}{ RT } & \multicolumn{3}{|l|}{ IKSI } \\
\hline & $M S E$ & $F$ & $\eta_{\mathrm{p}}^{2}$ & $M S E$ & $F$ & $\eta_{\mathrm{p}}^{2}$ \\
\hline Keyboard (K) & $1,744.0$ & 2.7 & .055 & 462.2 & $11.2^{*}$ & .196 \\
\hline $\mathrm{K} \times \operatorname{Exp}$ & & $<1$ & .000 & & $<1$ & .004 \\
\hline Echo (E) & $1,510.5$ & $10.8^{*}$ & .190 & 181.4 & $26.7^{*}$ & .358 \\
\hline $\mathrm{E} \times \operatorname{Exp}$ & & $<1$ & .014 & & $9.0^{*}$ & .163 \\
\hline $\mathrm{K} \times \mathrm{E}$ & 709.1 & $<1$ & .013 & 55.4 & $<1$ & .000 \\
\hline $\mathrm{K} \times \mathrm{E} \times \operatorname{Exp}$ & & $<1$ & .005 & & $<1$ & .000 \\
\hline
\end{tabular}

Degrees of freedom for each effect $=1,23 ;{ }^{*} p<.05$

3.1, $M S E=14,876.05, p=.085$. Thus, implicit error detection was not affected by the requirement to report errors explicitly.

\section{Conclusions}

Consistent with Experiment 1, posterror slowing was unaffected by visual feedback from the screen or the hands, suggesting that implicit error detection does not depend on visual feedback. Explicit error reports showed that typists had significantly higher percentages of detecting errors when keystrokes were echoed than when they were not. This finding suggests that visual feedback from the screen is an important source of information for explicit error detection (Logan \& Crump, 2010, 2011; Long, 1976; Rabbitt, 1978; Rieger, Martinez, \& Wenke, 2011). The fact that typists were able to detect errors in the absence of visual feedback from the screen suggests that explicit error detection can also utilize visual or kinesthetic feedback from the hands. However, the extended IKSIs that occurred in these instances indicate that when the outer loop monitors keystrokes, it must adjust the inner-loop timing parameters to slow keystroke execution (Snyder \& Logan, 2013).

\section{General discussion}

The present study investigated how the availability of visual feedback from the screen and hands influences the way that skilled typing is controlled. The two-loop theory of skilled typing (Logan \& Crump, 2009, 2011) states that an explicit outer processing loop generates words and monitors visual feedback from the screen to ensure that words are typed correctly. Indeed, we found that visual feedback from the screen did affect explicit error reports (Exp. 2). The twoloop theory also states that an implicit inner processing loop generates keystroke sequences and monitors kinesthetic feedback from the hands to ensure that keystrokes are executed correctly. Consistent with this tenet of the two-loop theory, we found that visual feedback from the screen and hands did not affect posterror slowing in either experiment. These findings are consistent with the many typing studies that have demonstrated dissociations between the outer and inner loops (e.g., Abrahamse et al., 2013; Logan \& Crump, 2009; Snyder \& Logan, 2013; Tapp \& Logan, 2011; Verwey, 1999).

The two-loop theory (Logan \& Crump, 2011) states that the inner loop is nested within the outer loop, so the outer loop dictates what the inner loop should do and sets restrictions for how it should be done. Indeed, previous studies have shown that the outer loop can adjust the parameters within which the inner loop must operate to adapt to task requirements (Logan \& Crump, 2009; Snyder \& Logan, 2013; Tapp \& Logan, 2011; Yamaguchi et al. 2013a, b). The present study extends these findings, demonstrating that the outer loop also adjusts inner-loop timing parameters to compensate for reduced visual feedback. In Experiments 1 and 2, RTs were longer when response echoes were absent than when they were present. We suggest that this finding reflects adjustments in the time allotted for the inner loop to generate motor programs more cautiously. In Experiment 2, IKSIs were longer when response echoes were absent than when they were present. We suggest that this finding reflects adjustments in the time allotted for the inner loop to produce keystrokes so that the outer loop could monitor them explicitly (Logan \& Crump, 2009; Snyder \& Logan, 2013; Tapp \& Logan, 2011). Feedback conditions were blocked, so typists knew what feedback would be available before they began each block. Therefore, we suggest that processing rate adjustments are indicative of a preemptive strategy rather than a reflexive calibration. Such an interplay between higher- and lower-level processes is an aspect of hierarchical control of skill that has been insufficiently investigated, so future investigations should focus more on this factor.

\section{Yesterday's professional typists and today's skilled typists}

The question of how visual feedback influences the control of skilled typing was investigated in the 1960s and 1970s (Diehl \& Seibel, 1962; Long, 1976; Rabbitt, 1978; West, 1967). The present study differed from these earlier studies in three respects: First, the earlier studies used a continuous typing task in which typing performance was only measured via IKSIs and error rates. IKSI reflects inner-loop processes more directly than outer-loop processes. As a result, measuring only IKSIs and error rates makes it difficult to separate the effects of visual feedback on outer- and inner-loop processes. Our experiments used a discrete typing task in which typing performance was measured by RTs, IKSIs, explicit error reports, and posterror slowing. This design allowed us to distinguish the effects of visual feedback on outer- and inner-loop processes: 
Analyzing RTs and IKSIs allowed us to distinguish control processes in the two loops. Analyzing explicit error reports and posterror slowing allowed us to distinguish monitoring processes in the two loops.

Second, we tested a different population of typists. The previous studies tested professional typists who were drawn from a special population. Professional typists' training emphasized not looking at their hands. We tested college students who were drawn from a larger population. Modern typists' training places less emphasis on not looking at their hands.

Third, our typists typed on different machines than the typists in previous studies. Modern keyboards require less force and shorter finger movements than the electric typewriters of the 1960s and 1970s and the mechanical typewriters of earlier decades. These differences change the haptic, kinesthetic, and proprioceptive feedback and might influence the relative importance of visual feedback.

Despite the many differences between modern typists and the professional typists of yesteryear, most of our findings were consistent with those of the classical studies. However, there was one notable exception. We found that removing visual feedback from the screen resulted in longer IKSIs. Classic typing studies had shown no such effect (Diehl \& Seibel, 1962; Long, 1976; West, 1967). Thus, modern typists may rely more on visual feedback than professional typists did. A possible explanation for this difference is that professional typists typed on typewriters, so the text they copied was separate from the text that they produced, and looking between documents could cause them to lose their place. Modern typists type on computers, where the texts they copy and produce can be close together, so there is no need to look away from the screen or the keyboard. Modern typists also compose text more often than they copy it (Logan \& Crump, 2011), and that may encourage them to keep their eyes on the screen to evaluate their composition as they type it. Hence, the results suggest that changes in the devices and purposes of typing have influenced how typing is controlled.

\section{Conclusions}

The present work has provided three novel contributions to the literature. First, it provides a two-loop theory account of the differential effects of visual feedback on the control of skilled typing. Second, it demonstrates that the availability of visual feedback may lead the outer loop to adjust the parameters within which the inner loop must operate. Third, it suggests that modern typists rely on visual feedback more than had the professional typists of yesteryear. The task of typing has remained largely unchanged since the 1900s, but the technology that typists interact with has evolved considerably. It appears that the cognitive system is able to adapt to the technological context by tailoring the ways in which it utilizes feedback to control skilled behavior, compensating for the available feedback to optimize performance.

Author note This research was supported by Grant Nos. BCS 0957074 and BCS 1257272 from the National Science Foundation.

\section{References}

Abrahamse, E. L., Ruitenberg, M. F., de Kleine, E., \& Verwey, W. B. (2013). Control of automated behavior: Insights from the discrete sequence production task. Frontiers in Human Neuroscience, 7(82), 1-16. doi:10.3389/fnhum.2013.00082

Crump, M. J. C., \& Logan, G. D. (2010a). Hierarchical control and skilled typing: Evidence for word-level control over the execution of individual keystrokes. Journal of Experimental Psychology: Learning, Memory, and Cognition, 36, 1369-1380. doi:10.1037/a0020696

Crump, M. J. C., \& Logan, G. D. (2010b). Warning: This keyboard will deconstruct - The role of the keyboard in skilled typewriting. Psychonomic Bulletin \& Review, 17, 394-399. doi:10.3758/PBR. 17.3.394

Crump, M. J. C., \& Logan, G. D. (2013). Prevention and correction in post-error performance: An ounce of prevention and a pound of cure. Journal of Experimental Psychology: General, 142, 692-709.

Davies, M. (2008). The Corpus of Contemporary American English (COCA): 410+ million words, 1990-present. Available online at www.americancorpus.org

Diehl, M. J., \& Seibel, R. (1962). The relative importance of visual and auditory feedback in skilled typewriting. Journal of Applied Psychology, 46, 365-369.

Fautrelle, L., \& Bonnetblanc, F. (2012). On-line coordination in complex goal-directed movements: A matter of interactions between several loops. Brain Research Bulletin, 89, 57-64. doi:10.1016/j. brainresbull.2012.07.005

Gordon, A. M., \& Soechting, J. F. (1995). Use of tactile afferent information in sequential finger movements. Experimental Brain Research, 107, 281-292.

Keele, S. W. (1968). Movement control in skilled motor performance. Psychonomic Bulletin \& Review, 70, 387-403.

Keele, S. W., Cohen, A., \& Ivry, R. (1990). Motor programs: Concepts and issues. In M. Jeannerod (Ed.), Attention and performance XIII: Motor representation and control (pp. 77-110). Hillsdale, NJ: Erlbaum.

Keele, S. W., \& Posner, M. I. (1968). Processing of visual information in rapid movements. Journal of Experimental Psychology, 77, 155158.

Lashley, K. S. (1951). The problem of serial order in behavior. In L. A. Jeffress (Ed.), Cerebral mechanisms in behavior (pp. 112-136). New York, NY: Wiley.

Logan, G. D., \& Crump, M. J. C. (2009). The left hand doesn't know what the right hand is doing: The disruptive effects of attention to the hands in skilled typewriting. Psychological Science, 20, 1296-1300. doi:10.1111/j.1467-9280.2009.02442.x

Logan, G. D., \& Crump, M. J. C. (2010). Cognitive illusions of authorship reveal hierarchical error detection in skilled typists. Science, 330, 683-686. doi:10.1126/science. 1190483

Logan, G. D., \& Crump, M. J. C. (2011). Hierarchical control of cognitive processes: The case for skilled typewriting. In B. H. Ross (Ed.), The psychology of learning and motivation: Advances in research and theory (Vol. 54, pp. 1-27). Burlington, MA: Academic Press. 
Logan, G. D., \& Zbrodoff, N. J. (1998). Stroop type interference: Congruity effects in color naming with typewritten responses. Journal of Experimental Psychology: Human Perception and Performance, 24, 978-992. doi:10.1037/0096-1523.24.3.978

Long, J. (1976). Visual feedback and skilled keying: Differential effects of masking the printed copy and the keyboard. Ergonomics, 19, 93-110.

Miller, G., Galanter, E., \& Pribram, K. (1960). Plans and the structure of behavior. New York, NY: Holt.

Rabbitt, P. M. A. (1966). Error correction time without external error signals. Nature, 212, 438. doi:10.1038/212438a0

Rabbitt, P. M. A. (1978). Detection of errors by skilled typists. Ergonomics, 21, 945-958. doi:10.1080/00140137808931800

Rieger, M., Martinez, F., \& Wenke, D. (2011). Imagery of errors in typing. Cognition, 121, 163-175. doi:10.1016/j.cognition.2011.07.005

Rosenbaum, D. A., Cohen, R. G., Jax, S. A., Weiss, D. J., \& van der Wel, R. (2007). The problem of serial order in behavior: Lashley's legacy. Human Movement Science, 26, 525-554.

Rumelhart, D. E., \& Norman, D. A. (1982). Simulating a skilled typist: A study of skilled cognitive-motor performance. Cognitive Science, 6, 1-36. doi:10.1207/s15516709 $\operatorname{cog} 0601 \_1$

Salthouse, T. A. (1986). Perceptual, cognitive, and motoric aspects of transcription typing. Psychological Bulletin, 99, 303-319. doi:10. 1037/0033-2909.99.3.303

Shaffer, L. H. (1976). Intention and performance. Psychological Review, 83, 375-393. doi:10.1037/0033-295X.83.5.375

Snyder, K. M., \& Logan, G. D. (2013). Monitoring-induced disruption in skilled typewriting. Journal of Experimental Psychology: Human Perception and Performance, 39, 1409-1420. doi:10.1037/ a0031007

Snyder, K. M., \& Logan, G. D. (2014). The problem of serial order in skilled typing. Journal of Experimental Psychology: Human Perception and Performance, 40, 1697-1717.
Sternberg, S., Knoll, R. L., \& Turock, D. L. (1990). Hierarchical control in the execution of action sequences: Tests of two invariance principles. In M. Jeannerod (Ed.), Attention and performance XIII: Motor representation and control (pp. 3-55). Hillsdale, NJ: Erlbaum.

Tapp, K. M., \& Logan, G. D. (2011). Attention to the hands disrupts skilled typewriting: The role of vision in producing the disruption. Attention, Perception, \& Psychophysics, 73, 2379-2383. doi:10. 3758/s13414-011-0208-5

Verwey, W. B. (1999). Evidence for a multistage model of practice in a sequential movement task. Journal of Experimental Psychology: Human Perception and Performance, 25, 1693-1708.

West, L. J. (1967). Vision and kinesthesis in the acquisition of typewriting skill. Journal of Applied Psychology, 51, 161-166.

Wilson, M. (1987). MRC Psycholinguistic Database: Machine usable dictionary (Version 2.00). Cambridge, UK: Medical Research Council. Available online at www.psy.uwa.edu.au/mrcdatabase/ uwa mrc.htm

Yamaguchi, M., Crump, M. J. C., \& Logan, G. D. (2013a). Speedaccuracy trade-off in skilled typewriting: Decomposing the contributions of hierarchical control loops. Journal of Experimental Psychology: Human Perception and Performance, 39, 678-699. doi:10.1037/a0030512

Yamaguchi, M., \& Logan, G. D. (2014). Pushing typists back on the learning curve: Revealing chunking in skilled typewriting. Journal of Experimental Psychology: Human Perception and Performance, 40, 592-612. doi:10.1037/a0033809

Yamaguchi, M., Logan, G. D., \& Li, V. (2013b). Multiple bottlenecks in hierarchical control of action sequences: What does "response selection" select in skilled typewriting? Journal of Experimental Psychology: Human Perception and Performance, 39, 1059-1084. doi:10.1037/a0030431 